\title{
GidA, a tRNA Modification Enzyme, Contributes to the Growth, and Virulence of Streptococcus suis Serotype 2
}

\author{
Ting Gao ${ }^{1,2,3}$, Meifang Tan ${ }^{1}$, Wanquan Liu ${ }^{1}$, Chunyan Zhang ${ }^{1}$, Tengfei Zhang ${ }^{2}$, \\ Linlin Zheng ${ }^{1}$, Jiawen Zhu ${ }^{1}$, Lu Li ${ }^{1,4}$ and Rui Zhou ${ }^{1,4 *}$ \\ 1 State Key Laboratory of Agricultural Microbiology, College of Veterinary Medicine, Huazhong Agricultural University, Wuhan, \\ China, ${ }^{2}$ Veterinary Medicine Laboratory, Institute of Animal Husbandry and Veterinary Science, Hubei Academy of \\ Agricultural Science, Wuhan, China, ${ }^{3}$ Wuhan Chopper Biology Co., Ltd., Wuhan, China, ${ }^{4}$ Cooperative Innovation Center of \\ Sustainable Pig Production, Wuhan, China
}

OPEN ACCESS

Edited by:

Matthew S. Francis,

Umeå University, Sweden

Reviewed by:

Michael Marceau,

University of Lille, France

Jesus Aranda,

Universitat Autònoma de Barcelona,

Spain

Amin A. Fadl,

University of Wisconsin-Madison, USA

*Correspondence:

Rui Zhou

rzhou@mail.hzau.edu.cn

Received: 02 February 2016 Accepted: 27 March 2016

Published: 19 April 2016

Citation:

Gao T, Tan M, Liu W, Zhang C, Zhang T, Zheng L, Zhu J, Li L and Zhou R (2016) GidA, a tRNA Modification Enzyme, Contributes to the Growth, and Virulence of Streptococcus suis Serotype 2. Front. Cell. Infect. Microbiol. 6:44. doi: 10.3389/fcimb.2016.00044
Glucose-inhibited division protein (GidA), is a tRNA modification enzyme functioning together with $\mathrm{MnmE}$ in the addition of a carboxymethylaminomethyl group to position 5 of the anticodon wobble uridine of tRNA. Here, we report a GidA homolog from a Chinese isolate SC-19 of the zoonotic Streptococcus suis serotype 2 (SS2). gidA disruption led to a defective growth, increased capsule thickness, and reduced hemolytic activity. Moreover, the gidA deletion mutant ( $\triangle$ gidA) displayed reduced mortality and bacterial loads in mice, reduced ability of adhesion to and invasion in epithelial cells, and increased sensitivity to phagocytosis. The iTRAQ analysis identified 372 differentially expressed (182 up- and 190 down-regulated) proteins in $\triangle$ gidA and SC-19. Numerous DNA replication, cell division, and virulence associated proteins were downregulated, whereas many capsule synthesis enzymes were upregulated by gidA disruption. This is consistent with the phenotypes of the mutant. Thus, GidA is a translational regulator that plays an important role in the growth, cell division, capsule biosynthesis, and virulence of SS2. Our findings provide new insight into the regulatory function of GidA in bacterial pathogens.

Keywords: Streptococcus suis (S. suis), glucose-inhibited division protein (GidA), tRNA modification, iTRAQ, growth, capsule synthesis, virulence

\section{INTRODUCTION}

Streptococcus suis is an important zoonotic pathogen causing lethal infections in humans and pigs (Lun et al., 2007). S. suis infection in human is an emerging public health issue, whereas that in pigs causes severe economic problems in the pig industry (Wertheim et al., 2009). Two large outbreak of human S. suis infections were reported in China in 1998 and 2005, resulting in 229 infections and 52 deaths (Yu et al., 2006; Lun et al., 2007). Among the 33 serotypes classified on the basis of antigenicity of capsular polysaccharide (CPS), S. suis serotype 2 (SS2) is the most virulent and prevalent strain isolated from diseased pigs (Smith et al., 1999). Several virulence-associated factors responsible for the pathogenecity of $S$. suis, such as muramidase-released protein, suilysin (Sly), extracellular factor, fibrinonectin- and fibrinogen-binding proteins, enolase, arginine deiminase system (ADS), 
and glyceraldehyde-3-phosphate dehydrogenase (GAPDH), were identified over the past decade (Jing et al., 2008; Feng et al., 2014).

S. suis infection is a major cause of meningitis, septicemia, and arthritis. S. suis primarily colonizes the palatine tonsils, which is one of its natural habitats, then breaches epithelial cell barriers, reaches the bloodstream, disseminates through the blood circulation system, and finally invades different organs of the host (Fittipaldi et al., 2012). In this process, many proteins are regulated (up-regulated or down-regulated) at the translation level in response to surroundings change and environmental signals. However, the regulatory mechanism of genes which are preferentially regulated by this pathogen during specific stages of host infection has not yet been clearly demonstrated. GidA-like proteins, functioning as a tRNA modification enzyme, are widely distributed in nature and conserved among eukaryotes and prokaryotes (Yim et al., 2006). GidA is a FAD-binding protein and, together with MnmE, catalyzes the addition of carboxymethylaminomethyl group at position 5 of the wobble uridine of tRNAs (Shi et al., 2009). This modification contributes to proper and efficient protein translation (Fislage et al., 2014). GidA and MnmE serve essentially to prevent premature translation termination resulting from $(+2)$ translational frameshifts (Brégeon et al., 2001). GidA protein plays a different role in many bacteria: in Escherichia coli, deletion of gidA affects cell division when it is grown on glucose (Von Meyenburg et al., 1982); in Streptococcus mutans, gidA is involved in survival under stress conditions ( $\mathrm{Li}$ et al., 2014); in Aeromonas hydrophila, gidA regulates virulence protein, cytotoxic enterotoxin (Sha et al., 2004); in Salmonella enterica, disruption of gidA affects cell division and regulates the virulence proteins (Shippy et al., 2012; Rehl et al., 2013); and in Pseudomonas syringae, gidA is a global regulator (Kinscherf and Willis, 2002). In fact, GidA can regulate the expression of multiple proteins at the level of translation through tRNA modification (Kinscherf and Willis, 2002; Yim et al., 2006), and thus can regulate the survival of bacteria under stress condition in response to environmental signals.

In our previous study, the gidA gene was identified to be up-regulated in the brains and lungs of $S$. suis infected pigs, suggesting that GidA may play a role during the infection ( $\mathrm{Li}$ et al., 2010). In this study, we identified a functional gene SSU05_2163, which is annotated as gidA in S. suis 05ZYH33 genome and encodes a tRNA modification enzyme. Our data demonstrate that GidA is a translational regulator that affects cell growth and division, capsule polysaccharide biosynthesis, and expression of virulence proteins in S.suis.

\section{MATERIALS AND METHODS}

\section{Bacterial Strains, Plasmids, and Growth Conditions}

The bacterial strains and plasmids used in this study are listed in Table 1. The SS2 strain SC-19 was isolated from a sick pig during an epidemic outbreak in Sichuan Province in China in 2005 ( Li et al., 2009). Bacterial solution for experiment was prepared as follows: SC-19 and $\Delta$ gidA were grown at $37^{\circ} \mathrm{C}$ either in tryptic
TABLE 1 | Summary of bacterial strains and plasmid used in this study.

\begin{tabular}{|c|c|c|}
\hline $\begin{array}{l}\text { Strain or } \\
\text { plasmid }\end{array}$ & Characteristics and function ${ }^{a}$ & Sources or references \\
\hline \multicolumn{3}{|c|}{ BACTERIAL STRAINS } \\
\hline SC-19 & S. suis serotype 2, wide- type $\left(\right.$ Strep $\left.^{r}\right)$ & Li et al., 2009 \\
\hline$\Delta$ gidA & SC-19 gidA::erm (Strep ${ }^{r}$ Erm $\left.{ }^{r}\right)$ & This study \\
\hline E. coli $\mathrm{DH} 5 \alpha$ & Cloning host for recombinant vector & Trans \\
\hline \multicolumn{3}{|l|}{ PLASMID } \\
\hline pAT18 & $\begin{array}{l}\text { Contains an } \mathrm{Erm}^{\mathrm{r}} \text { gene expressing } \\
\text { erythromycin resistance rRNA methylase }\end{array}$ & Trieu-Cuot et al., 1991 \\
\hline pET28a & Expression vector; Kan ${ }^{r}$ & Novagen \\
\hline pSET4s & E. coli- S. suis shuttle vector; $\mathrm{Spc}^{r}$ & Takamatsu et al., 2001 \\
\hline pSET4s-G & $\begin{array}{l}\text { Derived from pSET4s used to knock out } \\
\text { gidA in SC-19; } \text { Spc }^{r} \text { Erm }^{r}\end{array}$ & This study \\
\hline
\end{tabular}

${ }^{\text {aStrep }}{ }^{r}$,streptomycin resistant; Erm ${ }^{r}$, erythromycin resistant; Kan ${ }^{r}$, kanamycin; Spcr spectinomycin, resistant.

soy broth, or on tryptone soy agar (Difco, France) containing $10 \%$ fetal bovine serum (Sijiqing, Hanzhou, China), and then the cultures were centrifuged and washed thrice with saline. To be consistent with the isobaric tags for relative and absolute quantitation (iTRAQ) analysis, all of the bacterial pellets were resuspended in the chemically defined medium (CDM) (Van De Rijn and Kessler, 1980) supplemented with 1\% glucose and grown to mid-log phase at $37^{\circ} \mathrm{C}$. E. coli $\mathrm{DH} 5 \alpha$ was used as host strain for cloning and maintained in Luria-Bertani (LB) broth (Difco) or plated on $\mathrm{LB}$ agar at $37^{\circ} \mathrm{C}$. The antibiotic concentration prepared for wild-type SC-19 was $20 \mu \mathrm{g} / \mathrm{ml}$ streptomycin. The antibiotic concentrations used to select the mutant strain $\Delta$ gidA were $100 \mu \mathrm{g} / \mathrm{ml}$ for spectinomycin and $90 \mu \mathrm{g} / \mathrm{ml}$ for erythromycin. The kanamycin concentration used to select $E$. coli strains harboring the plasmid of interest was $25 \mu \mathrm{g} / \mathrm{ml}$.

\section{Knockout of gidA}

To construct a $\triangle$ gidA mutant strain, we used the thermosensitive suicide vector pSET4s to delete gidA through homologous recombination as previously described (Takamatsu et al., 2001). Primers used in this study were designed according to the genome sequence of $S$. suis 05ZYH33 (GenBank accession number CP000407; Table 2). Two pairs of specific primers, Gup-F/Gup-R and Gdown-F/Gdown-R were used to clone the gidA upstream and downstream of the homologous regions into pSET4s. The $\mathrm{erm}^{\mathrm{r}}$ expression cassette was amplified from pAT18 by using primers Erm-F/Erm-R and then inserted between the upstream and downstream homologous arms in the recombinant pSET4s to generate the gidA-knockout vector pSET4s-G.

To obtain isogenic mutant $\triangle$ gidA, we electro transformed pSET4s-G plasmid into SC-19 competent cells (Zhang et al., 2012). The mutant strain was screened on TSA plates owing to its sensitivity to spectinomycin and resistance to erythromycin. To confirm the mutant, we amplified gidA through PCR by using the primers GidA-F/GidA-R.

\section{RNA Extraction and RT-PCR}

To confirm the mutant strain $\Delta g i d A$, we performed RT-PCR according to our previously reported methods (Tan et al., 2015). 
TABLE 2 | Primers used for PCR amplification and detection.

\begin{tabular}{|c|c|c|}
\hline Primers & Primers sequence $\left(5^{\prime}-3^{\prime}\right)^{\mathrm{a}}$ & Amplification for \\
\hline Gup-F & СTTCAAGCTTGCTTTGTGGACTTA & $\begin{array}{l}\text { Upstream border of } \\
\text { gidA }\end{array}$ \\
\hline Gup-R & GTTGTCGACTCATGTTGTTCTCTCCT & \\
\hline Gdown-F & GATCCCGGGGGCTGTTCTITCGC & $\begin{array}{l}\text { Downstream border of } \\
\text { gidA }\end{array}$ \\
\hline Gdown-R & ССССGАATTCTTCCTTGACСАCAACC & \\
\hline Erm-F & GTCTGGATCCCTTAGAAGCAAACTTAA & $\mathrm{Erm}^{r}$ gene \\
\hline Erm-R & GTTAGGATCCATCGATACAAATTCCCCG & \\
\hline GidA-F & CGGGATCCATGACACACACATTTGCAGA & gidA gene \\
\hline GidA-R & CGCTCGAGTTAGTGACTGTCCTTIGATT & \\
\hline $2162-\mathrm{F}$ & GTGATGAAAAGATTTCGATT & $\begin{array}{l}\text { Downstream gene of } \\
\text { gidA }\end{array}$ \\
\hline $2162-\mathrm{R}$ & TTATCCAAAGTCAAGCCA & \\
\hline $2164-F$ & GGTTGATTATAAAAGATGG & Upstream gene of gid $A$ \\
\hline $2164-\mathrm{R}$ & TCATGTTGTTCTCTCCTT & \\
\hline
\end{tabular}

a Underlined nucleotides denote enzyme restriction sites.

Briefly, RNA was isolated using SV Total RNA Isolation System (Promega, USA) according to the manufacturer's instructions. In addition, cDNA was synthesized using HiScript Q Select RT SuperMix (Vazyme, China) according to the manufacturer's instructions.

To confirm whether the upstream and downstream genes of gidA are unaffected and functioning normally, we designed the primers of SSU05_2162, gidA, and SSU05_2164 for RT-PCR (Table 2) from the cDNA.

\section{Western Blot}

To further confirm the mutant strain $\Delta g i d A$, we performed Western blot analysis according to our previously reported methods (Tan et al., 2015). Mouse anti-GidA serum was produced as described previously (Li et al., 2011) by using recombinant GidA protein. The PVDF membranes (Invitrogen, USA) were probed with primary antibodies against GidA (1:1000) or 3-phosphoglycerate kinase (PGK) (1:5000; Invitrogen) (Zhang et al., 2014). After washing, the membranes were incubated with goat anti-mouse IgG $(\mathrm{H}+\mathrm{L})-\mathrm{HPR}$ (1:5000; Southern Biotech, USA). Antibody-tagged protein bands were detected by using Western ECL Substrate Kit (Bio-Rad, USA).

\section{Transmission Electron Microscopy (TEM)}

To obtain an overview of the morphology of SC-19 and $\Delta$ gidA, we performed TEM as described previously (Zheng et al., 2011). The samples grown in CDM were harvested at mid-log phase and fixed with $2.5 \%$ glutaraldehyde overnight. The samples were then treated with $2 \%$ osmium tetroxide for $2 \mathrm{~h}$ and dehydrated in a serial dilution of ethanol. The dehydrated cells were embedded in epoxy resin and cell morphology was observed using an $\mathrm{H}$ 7650 TEM (HITACHI, Ltd., Tokyo, Japan). 20 bacterial cells were randomly chosen from the TEM micrographs to measure the thickness of capsule by using the software Image J, and then statistically analyzed on GraphPad prism 5.

\section{Hemolysin Assay}

Hemolysin activity was tested as described previously (Jacobs et al., 1994) with some modifications. Briefly, S. suis strains were grown in CDM up to mid-log phase, and the culture supernatant was collected by centrifugation at $12000 \mathrm{~g}$ for 2 min. The test samples $(100 \mu \mathrm{l})$ were incubated with $2 \%$ sheep erythrocyte suspension $(100 \mu \mathrm{l})$ in saline for $2 \mathrm{~h}$ at $37^{\circ} \mathrm{C}$ and $\mathrm{CDM}$ was used as negative control. Unlysed erythrocytes were centrifuged at $1500 \mathrm{~g}$ for $15 \mathrm{~min}$, and $100 \mu \mathrm{l}$ supernatant was transferred into a new plate (Jet Biofil, China). Absorption was subsequently measured at $550 \mathrm{~nm}$ by using a microELISA reader (Biotek, Vermont, USA).

\section{Mouse Infection Experiments}

This study was performed in accordance with the Guide for the Care and Use of Laboratory Animals Monitoring Committee of Hubei Province, China, and the protocol was approved by the Committee on the Ethics of Animal Experiments of the College of Veterinary Medicine, Huazhong Agricultural University. All efforts were made to minimize the suffering of the animals used in the study.

To detect the role of GidA in S. suis virulence, we divided 30 6-week-old female specific-pathogen-free (SPF) Kun-Ming mice into three groups (10 mice per group). Groups 1 and 2 were inoculated via intraperitoneal injection with $3 \times 10^{9} \mathrm{CFU}$ of either SC-19 (the $\mathrm{LD}_{50}$ for mice is $1.5 \times 10^{9} \mathrm{CFU}$ ) or $\Delta$ gidA. Saline was applied in Group 3 as negative control. The mice were observed for 7 days to obtain steady survival curves.

To detect the role of GidA on colonization in different organs, we performed murine colonization assay as described previously (Marion et al., 2011). A total of 15 6-week-old female SPF KunMing mice were inoculated intraperitoneally with $1 \times 10^{8} \mathrm{CFU}$ of a 1:1 mixture of mid-log phase SC-19 and $\Delta$ gidA. Saline was applied as negative control in five mice. At $12 \mathrm{~h}, 1$ day, and 3 days post infection (dpi), brain, lung, and spleen were obtained from five mice. The samples were homogenized after weighing, and serial dilutions were plated onto TSA agar. To count the colonies, we used $20 \mu \mathrm{g} / \mathrm{ml}$ streptomycin for SC-19, whereas $20 \mu \mathrm{g} / \mathrm{ml}$ streptomycin and $90 \mu \mathrm{g} / \mathrm{ml}$ erythromycin were used for $\Delta \operatorname{gid} A$.

\section{Phagocytosis Assay}

To probe the resistance of each strain to phagocytosis, we performed an experiment as described previously (Li et al., 2013). RAW264.7 cells were scraped up and resuspended in antibioticsfree medium. After adhering to six-well cell culture plate (Falcon, USA), the cells were infected with SS2 at mid-log phase to reach a ratio of 10 bacteria per macrophage (MOI $=10: 1)$. Phagocytosis proceeded for $30 \mathrm{~min}$ at $37^{\circ} \mathrm{C}$ and then the cells were washed with PBS thrice. The cells were incubated in medium containing penicillin $(100 \mu \mathrm{g} / \mathrm{ml})$ for $1 \mathrm{~h}$ at $37^{\circ} \mathrm{C}$ to kill extracellular bacteria. The culture supernatant was plated on TSA plates to confirm whether the antibiotics effectively killed the extracellular bacteria. The macrophages were then lysed in $1 \mathrm{ml}$ of sterile distilled water. Viable intracellular bacteria were determined by plating a serial dilution of the lysates on TSA agar. 


\section{Adhesion and Invasion Assays}

To evaluate the adhesion and invasion capacity of each strain, we performed an experiment as described previously (Ferrando et al., 2014). For the adherence assay, HEp-2 cells were infected with SS2 at mid-log phase to reach a ratio of 100 bacteria per cell $(\mathrm{MOI}=100: 1)$ and then incubated for $30 \mathrm{~min}$ at $37^{\circ} \mathrm{C}$. The monolayers were washed with PBS thrice and lysed in $1 \mathrm{ml}$ of sterile distilled water. Adherent bacteria (cell-associated bacteria) were determined by plating a serial dilution of the lysates on TSA agar. For invasion assay, the cells were incubated with bacteria for $2 \mathrm{~h}$ to allow invasion. The cells were subsequently incubated in medium containing penicillin $(100 \mu \mathrm{g} / \mathrm{ml})$ for $2 \mathrm{~h}$ to kill extracellular and surface-adherent bacteria. The monolayers were washed with PBS thrice and lysed in $1 \mathrm{ml}$ of sterile distilled water. Invasive bacteria (intracellular bacteria) were determined by plating a serial dilution of the lysates on TSA agar.

\section{Protein Extraction, Digestion, and Labeling with iTRAQ Reagents}

SC-19 and $\triangle$ gidA cells at mid-log phase were cultured in CDM as described above. Three independent biological replicates were homogenized in liquid nitrogen and then precipitated using trichloroacetic acid and acetone. The pellets were suspended in lysis buffer (4\% SDS, 100 Tris-HCl, and 1 mMDTT; pH7.6) and heated for $10 \mathrm{~min}$ at $100^{\circ} \mathrm{C}$. The cell suspensions were sonicated for $5 \mathrm{~min}$ ( $10 \mathrm{~s}$ sonication with $15 \mathrm{~s}$ interval) on ice and then protein concentration in supernatants was determined through Bradford protein assay. Each sample $(200 \mu \mathrm{g})$ was digested with $3 \mu \mathrm{g}$ of trypsin (Sigma, USA) at $37^{\circ} \mathrm{C}$ for $16 \mathrm{~h}$. iTRAQ labeling was performed according to the manufacturer's protocol (Applied Biosystems, Foster City, CA, USA). Briefly, each ITRAQ reagent was dissolved in $70 \mu \mathrm{l}$ of ethanol and added into the peptide mixture, respectively. After incubation for 2 $\mathrm{h}$ at room temperature, the reaction was quenched by adding $0.5 \%$ formic acid. iTRAQ tags were labeled as follows: the three SC-19 samples were labeled with iTRAQ 114, iTRAQ 115, and iTRAQ 116; and the three $\triangle$ gidA samples were labeled with iTRAQ 117, iTRAQ 118, and iTRAQ 119. The labeled peptides were combined and fractionated by using strong cation exchange (SCX) chromatography.

\section{LC-MS/MS Analysis}

After separation by SCX chromatography on an AKTA purifier 100 (GE Healthcare, USA), equal amounts of digested protein were loaded into a Thermo Scientific EASY column $\left(2 \mathrm{~cm}^{*} 100 \mu \mathrm{m} 5 \mu \mathrm{m}-\mathrm{C} 18\right)$ and then washed with solvent A $\left(99 \% \mathrm{H}_{2} \mathrm{O}\right.$, and $0.1 \%$ formic acid). By applying solvent $\mathrm{B}$ ( $84 \%$ acetonitrile, $16 \% \mathrm{H}_{2} \mathrm{O}$, and $0.1 \%$ formic acid), the peptides were eluted from the trapping column over a Thermo scientific EASY column $\left(75 \mu \mathrm{m}^{*} 100 \mathrm{~mm} 3 \mu \mathrm{m}-\mathrm{C} 18\right)$ with a gradient (0-45\% B for $100 \mathrm{~min}$ at $250 \mathrm{nl} / \mathrm{min}, 35-100 \%$ B for $8 \mathrm{~min}$, $100 \%$ B for $12 \mathrm{~min}$ ) using Thermo scientific Easy nLC system. MS/MS was carried out with a Q-Exactive mass spectrometer (Thermo Finnigan, USA) setting in a positive ion mode and data-dependent manner choosing the most abundant precursor ions with a full MS scan from 300 to $1800 \mathrm{~m} / z$, resolution of 70,000 at $m / z 200$. Determination of the target value was based on automatic gain control (AGC). Dynamic exclusion duration was $40 \mathrm{~s}$. MS/MS scan was acquired at a resolution of 17,500 at $\mathrm{m} / z$ 200. Normalized collision energy was $30 \mathrm{eV}$ and the under fill ratio was set at $0.1 \%$. Quantitation achieved by comparison of the peak areas and resultant peak ratios for either four MS/MS reporter ions, which range from 114 to $117 \mathrm{Da}$, or eight MS/MS reporter ions, which range from $113-119$ to $121 \mathrm{Da}$.

\section{Proteomic Data Analysis}

The acquired raw MS/MS data files were processed by Proteome Discoverer 1.4 (Thermo Scientific, USA) and searched by Mascot 2.2 (Matrix Science, Boston, MA) against the uniprot_Streptococcus_suis_23318_20150708.fasta (23,318 sequence, downloaded July 8,2015$)$. The search was conducted by applying trysin as a specific enzyme and the parameters used for normal peptides were as follows: peptide mass tolerance: 20 ppm, fragment mass tolerance: $0.1 \mathrm{Da}$, max missed cleavages: 2, fixed modifications: carbamidomethyl (C), iTRAQ8plex(K), and iTRAQ8plex(N-term), variable modifications: oxidation (M), database pattern: decoy, false-discovery rate $(\mathrm{FDR}) \leq$ 0.01 (Sandberg et al., 2012). Each of the confident protein identification involved at least one unique peptide. The quantitative protein ratios were weighed and normalized by the protein median ratio in Mascot. To evaluate the differentially expressed (DE) proteins between $\triangle$ gidA and SC-19, we use the fold change of $>1.2$ or $<0.8333$ and FDR of $<0.05$ to represent up- or down-regulation.

\section{Statistical Analysis}

Unless otherwise specified, the data were analyzed using twotailed, unpaired $t$-tests and all experiments were performed in triplicate at least thrice. All of the data were expressed as mean standard errors of the means (SEM), and $p<0.05$ is the threshold for significance. Statistical analysis was performed on GraphPad prism 5.

\section{RESULTS}

\section{Construction and Characterization of $\triangle$ gidA}

The colonies sensitive to spectinomycin and resistant to erythromycin were selected as candidates of gidA deletion mutants, which were confirmed by PCR (Figure 1A), RT-PCR (Figure 1B), and Western blot analysis (Figure 1C). The colonies of $\triangle$ gidA appeared smaller than those of SC-19 when cultured on TSA plates overnight (Figure 2A). The growth curves showed that $\triangle$ gidA grew slower in the CDM than SC-19 (Figure 2B). However, no obvious difference in CFU counts was observed during the initial $3 \mathrm{~h}$ of growth. TEM revealed that the mean capsule was significantly thicker in $\triangle \operatorname{gidA}(118 \pm 5 \mathrm{~nm})$ than in SC-19 (54 \pm 3 nm; $p<0.001$; Figure 2C).

\section{Reduced Hemolytic Activity}

The hemolysin assay showed that the hemolytic activity of $\Delta$ gidA was significantly reduced compared to that of SC-19 (Figure 2D), indicating that GidA could regulate the hemolytic activity of S. suis. 

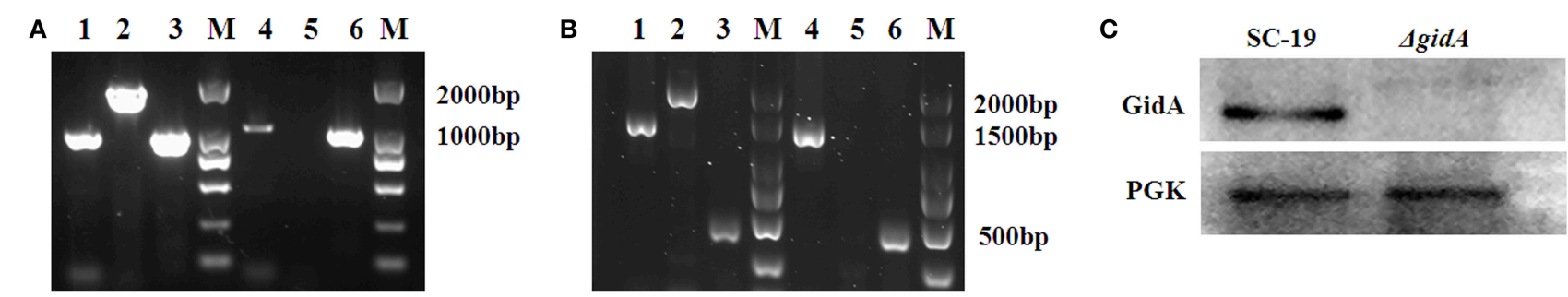

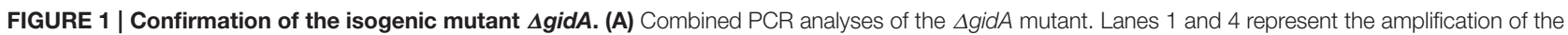
upstream border of gidA using the primer set Gup-F and Gup-R. Lanes 2 and 5 represent the amplification of gidA using the primer set GidA-F and GidA-R. Lanes 3 and 6 represent the amplification of the downstream border of gidA using the primer set Gdown-F and Gdown-R. Lanes 1-3 use genomic DNA of SC-19 as templates, whereas Lanes 4-6 use genomic DNA of $\triangle$ gidA as templates. (B) Confirmation of the $\triangle$ gidA mutant by RT-PCR. Lanes 1 and 4 represent the amplification of downstream gene of gidA using the primer set 2162-F and 2162-R. Lanes 2 and 5 represent the amplification of gidA using primer set GidA-F and GidA-R. Lanes 3 and 6 represent the amplification of upstream gene of gidA using the primer set 2164-F and 2164-R. Lanes 1-3 use cDNA of SC-19 as templates, whereas Lanes 4-6 use cDNA of $\triangle$ gidA as templates. (C) Confirmation of the $\triangle$ gidA mutant by Western blot analysis. The supernatant of cell lysate from SC-19 and $\triangle$ gidA was disposed for immunoblot analysis with GidA or PGK polyclonal antibodies. An antibody directed against PGK was used as loading control.
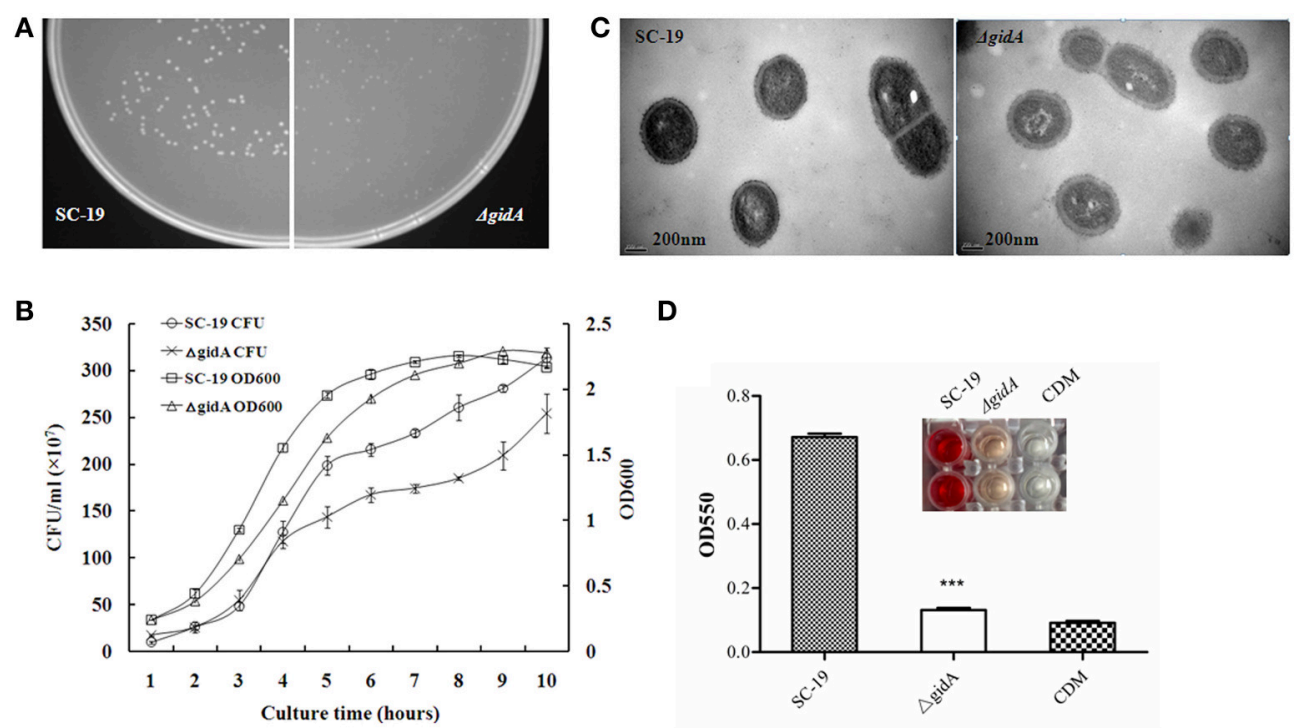

D

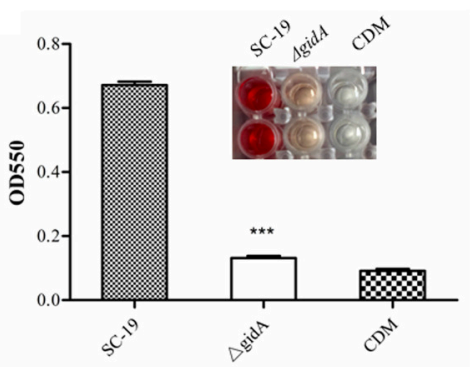

FIGURE 2 | Characterization of gidA mutant. (A) SC-19 and $\triangle$ gidA mutant cultured overnight at $37^{\circ} \mathrm{C}$ on TSA plates. (B) Bacterial cell density was measured spectrometrically at $600 \mathrm{~nm}$, and separate aliquots of the bacterial suspensions were serially diluted and plated to determine CFU numbers per milliliter. Data were collected at the indicated times. (C) Transmission electron micrographs of bacteria; the bars represent $400 \mathrm{~nm}$ ( $\left.{ }^{* * *} p<0.001\right)$. (D) Microplate showing hemolytic activity of the supernatants collected from SC-19 and $\Delta$ gidA mutant grown in CDM. Absorption was measured at $550 \mathrm{~nm}$ to determine suilysin production $\left({ }^{* * *} p<0.001\right)$. CDM was used as negative control.

\section{Attenuated Virulence and Decreased Bacterial Loads in Mice}

Mice were experimentally infected to detect the role of gidA in S. suis virulence. All of the SC-19-infected mice displayed severe clinical symptoms, such as septicemia and meningitis during $1 \mathrm{dpi}$, and most of the infected mice (9/10) died during the 7 day observation period. By contrast, the $\Delta g i d A$-infected mice exhibited more slight clinical symptoms and low mortality $(2 / 10)$ (Figure 3A). Therefore, $\Delta$ gidA virulence is markedly attenuated.

To better evaluate the pathogenecity of $\Delta g i d A$, we performed a colonization experiment by using intraperitoneal route of inoculation. Bacteria were recovered from brains, lungs, and spleens at different time points post infection. The bacterial loads in brain, lung, and spleen were much lower in $\triangle g i d A$ than SC-19 from $12 \mathrm{~h}$ to $3 \mathrm{dpi}$, and the mutant strain was almost cleared at the 3 dpi (Figures 3B-D).

\section{Greater Sensitivity to Phagocytosis by RAW264.7 Cells}

To investigate the role of gidA on phagocytosis of S. suis, we performed a phagocytosis assay by using RAW264.7 cells. The numbers of intracellular bacteria for $\Delta$ gidA $(34,320 \pm 3130$ $\mathrm{CFU} /$ well) were approximately two fold higher than those of SC19 (18430 $\pm 821 \mathrm{CFU} /$ well; $p<0.001$; Figure 4A). This result 

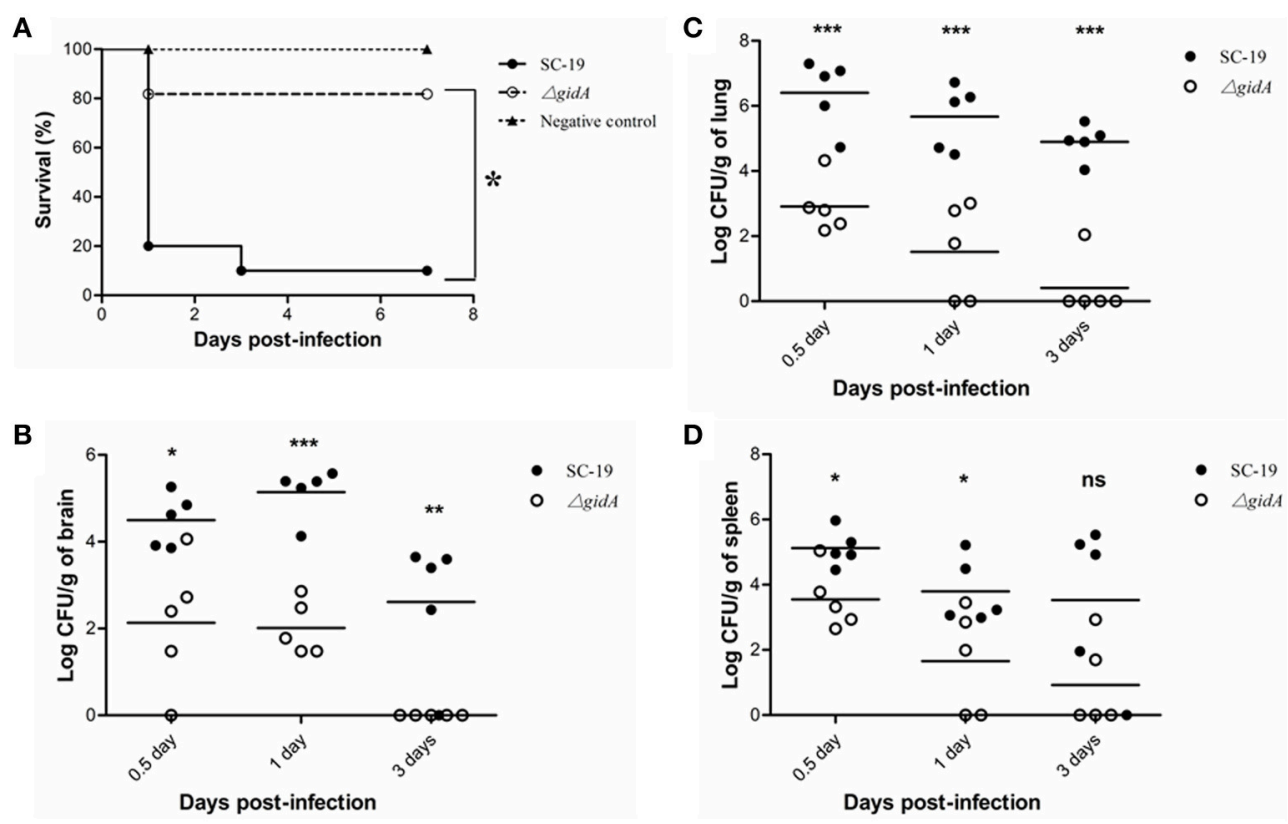

FIGURE 3 | Mouse infection experiments. (A) Survival curves for mice in experiment infection. Ten mice in each group were separately injected intraperitoneally i with $3 \times 10^{9} \mathrm{CFU} /$ mice of SC-19 and $\Delta$ gidA. Ten mice were inoculated with saline and served as negative control. Significant difference in survival between different groups were analyzed by Log Rank test $(p<0.05)$. (B) Bacteria loads in (B) brain, (C) lung, and (D) in spleen. The SC-19 and $\Delta$ gidA mutant strains were distinguished by erythromycin added in the TSA plates. Statistical significance was determined by two-tailed $t$-test (ns, $\left.p>0.05 ;{ }^{*} p<0.05 ;{ }^{* *} p<0.01 ;{ }^{* * *} p<0.001\right)$.
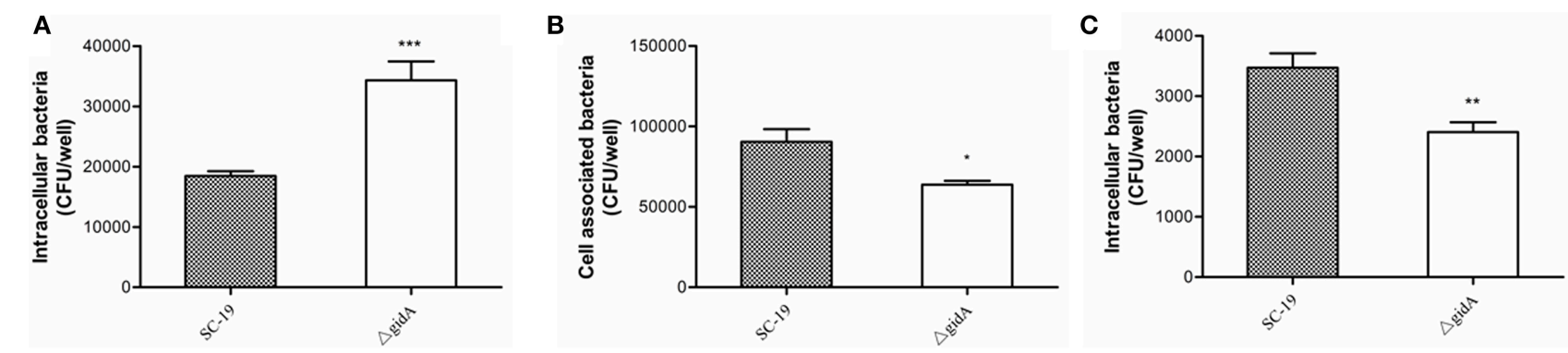

FIGURE 4 | Phagocytosis, adhesion, and invasion assays. (A) Phagocytosis of S. suis by murine macrophages. SC-19 and $\triangle$ gidA mutant were incubated with RAW264.7 cells for $30 \mathrm{~min}$ at a multiplicity of infection of $10(\mathrm{MOI}=10: 1)$. Penicillin $(100 \mu \mathrm{g} / \mathrm{ml})$ was then used to kill the extracellular bacteria. The mutant strain $\Delta$ gidA showed significantly reduced anti-phagocytosis capability compared with SC-19 $\left.{ }^{* * *} p<0.001\right)$. (B) The mutant strain $\Delta$ gidA showed significantly reduced levels of adherence to HEp-2 cells compared with the $=$ degree of adherence of SC-19 ( $\left.{ }^{*} p<0.05\right)$. (C) The mutant strain $\Delta$ gidA showed significantly reduced levels of invasion of HEp-2 cells compared with that of SC-19 $\left(^{* *} p<0.01\right)$.

indicates that inactivation of gidA can impair the capacity of S.suis to resist phagocytosis by macrophages.

\section{Impaired Adhesion and Invasion Capability to Epithelial Cells}

The adhesion assay revealed that the numbers of cell-associated bacteria of $\Delta \operatorname{gidA}(63,800 \pm 2437 \mathrm{CFU} /$ well $)$ were significantly lower than those of SC-19 (90,400土7891 CFU/well; $p<0.05$; Figure 4B). In the invasion assay, the numbers of the cell intracellular bacteria of $\triangle$ gidA (2406 $\pm 163 \mathrm{CFU} /$ well $)$ were significantly lower than those of SC-19 (3470 $\pm 241 \mathrm{CFU} /$ well; $p<0.01$; Figure 4C). These results suggest that deletion of gidA impairs the ability of S. suis to adhere to and invade in epithelial cells.

\section{Analysis of the DE Proteins}

The iTRAQ labeling was used to identify the DE proteins in SC-19 and $\triangle$ gidA. A total of 1449 proteins were detected and quantified, 372 of which were DE proteins, including 182 upregulated and 190 down-regulated proteins (Table S1).

These DE proteins were annotated using Blast2GO according to biological process, molecular function, and cellular component (Figure 5). In terms of biological process, the $372 \mathrm{DE}$ proteins were classified into 11 categories. The top categories with the highest number of DE proteins were metabolic process 


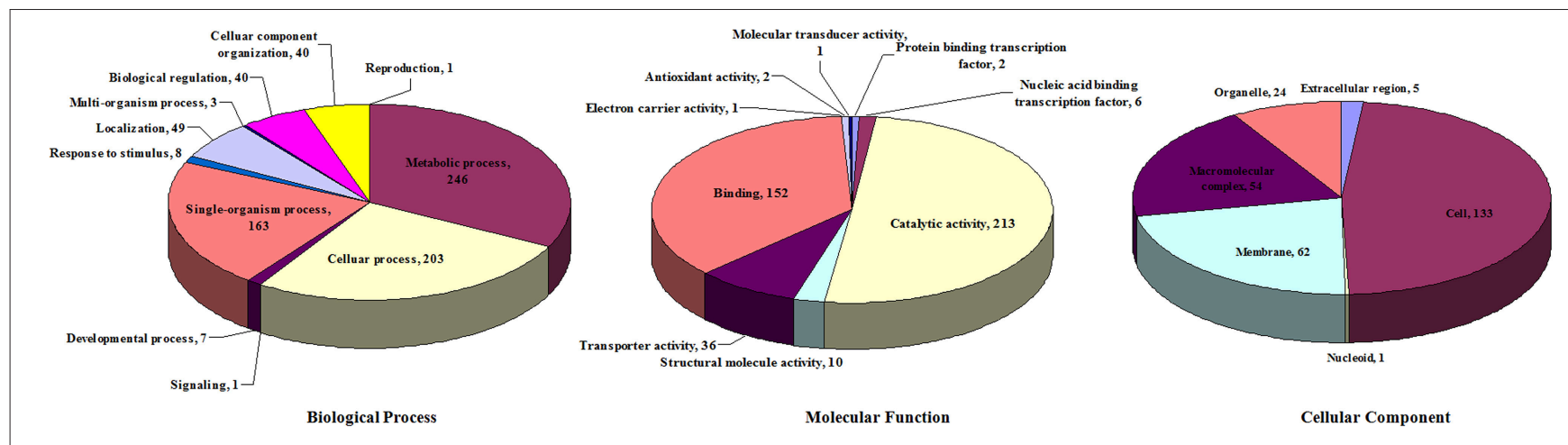

FIGURE 5 | Distribution of differentially expressed proteins according to GO annotation.

(246, 66.1\%), cellular process $(203,54.6 \%)$, and single-organism process $(163,43.8 \%)$; these three functional categories are the most important in $S$. suis response to environmental stresses. In terms of molecular function, the $372 \mathrm{DE}$ proteins were classified into nine categories. The top two categories with the highest number of DE proteins were catalytic activity $(213,57.3 \%)$ and binding $(152,40.9 \%)$. In terms of cellular component, the 372 DE proteins were classified into six categories. The top three categories with the highest number of DE proteins were cell (133, $35.8 \%)$, membrane $(62,16.7 \%)$, and macromolecular complex $(54,14.5 \%)$.

\section{Proteins Involved in Growth and Cell Division}

Many growth- and cell division-associated proteins were regulated in the mutant strain (Table 3). Among these DE proteins, all of the DNA replication-, recombination- and repairrelated proteins, including DNA primase RnmV, DNA gyrase (GyrA and GyrB), superfamily I DNA/RNA helicase (PcrA), site-specific recombinases (XerS andXerD), DNA repair ATPase $(\mathrm{RecN})$, DNA recombination protein $(\mathrm{RmuC})$, ribonucleases (RnhB, RNase H, G, and E), and 3-methyladenine DNA glycosylase (Tag), were down-regulated. The other DE proteins, including DivIVA, FtsQ, FtsX, FtsI, GpsB, StpK, PhpP, Cps2C, and MurD, are involved in cell division. Except for FtsX and $\mathrm{GpsB}$, all other proteins were down-regulated.

\section{Proteins Involved in CPS Synthesis}

Except for Cps2C, the five enzymes involved in CPS synthesis were up-regulated in the mutant strain (Table 3). The induced enzymes include rhamnosyltransferase Cps2F, sialic acid synthase Cps2P, UNP- $N$-acetyglucosamine 2-epimerase Cps2Q, acetyltransferase $\mathrm{Cps} 2 \mathrm{R}$, and $\mathrm{CMP}-\mathrm{N}$-acetylneuraminic acid synthetase Cps2S.

\section{Proteins Involved in Virulence}

Several virulence factors were down-regulated in the mutant strain (Table 3). These factors include Sly, enolase, GAPDH, ADS (ArcABC), D-alanine-poly(phosphoribitol) ligase subunit 1 (DltA), glutamine synthetase (GlnA), glycosidase (GtfA), inosine $5^{\prime}$ - monophosphate dehydrogenase (IMPDH), adenylosuccinate synthase (PurA), and translation initiation factor 2 GTPase (SadP).

\section{DISCUSSION}

The tRNA modification enzyme GidA contributes to proper folding and stability of tRNA and to the correct interaction between codon and anticodon during translation in eukaryotes and prokaryotes (Fislage et al., 2014). GidA acts as a regulator for protein expression either by its direct effects on translation efficiency of particular gene products or through its broader effects transmitted via expression of regulators (Kinscherf and Willis, 2002). In several pathogenic bacteria, GidA is considered to play roles in many particular cellular processes such as growth, cell division, and virulence regulation (Shippy et al., 2011). However, its functions are not always the same in different bacterial species. S. suis is an important zoonotic pathogen, and the role of GidA in S. suis is unclear. Our study demonstrated that GidA could regulate not only growth, cell division, and capsule synthesis but also virulence of this important pathogen.

First, a gidA deletion mutant was constructed. We found that the mutant $\triangle$ gidA grew much slower than the parental strain SC19 on the TSA plate, and the size of the colonies of $\triangle$ gidA were obviously smaller than those of SC-19. The growth curves also confirmed the slow growth rate of the mutant strain. These results indicated that GidA can regulate $S$. suis growth. This finding agrees with the previous reports on E. coli (Von Meyenburg et al., 1982) and S. enterica (Rehl et al., 2013). To further understand the reason behind growth regulation, we performed a proteomics study. Numerous growth- and cell divisionassociated proteins were down-regulated by gidA disruption (Table 3). These proteins are classified into two classes: (i) DNA replication-, recombination-, and repair -related proteins, such as DNA primase (RnmV), DNA gyrase (GyrA and GyrB), DNA/RNA helicase (PcrA), site-specific recombinases (XerS and XerD), DNA repair ATPase $(\mathrm{RecN}), \mathrm{DNA}$ recombination protein ( $\mathrm{RmuC}$ ), ribonucleases ( $\mathrm{RnhB}, \mathrm{RNase} \mathrm{H}, \mathrm{G}$, and $\mathrm{E}$ ); (ii) cell division-related proteins, including DivIVA, FtsQ, FtsI, StpK, PhpP, Cps2C, and MurD, which positively regulate cell division (Dinardo et al., 1982; Edwards and Errington, 1997; Ferreira et al., 2003; Janto et al., 2011; Šink et al., 2013; Fleurie et al., 
TABLE 3 | Differentially expressed proteins associated with cell growth and division, capsule synthesis, and virulence.

\begin{tabular}{|c|c|c|c|c|c|}
\hline Protein name & Locus & Functions & Ratio( $\Delta$ gidA/SC-19) & Peptides & Sequence coverage (\%) \\
\hline \multicolumn{6}{|c|}{ CELL GROWTH AND DIVISION } \\
\hline Cps2C & SSU05_0566 & Tyrosine-protein kinase Wze & 0.4555 & 1 & 28.57 \\
\hline DivIVA & SSU05_0487 & Cell division initiation protein & 0.8229 & 1 & 59.83 \\
\hline- & SSU05_0133 & Adenine-specific DNA methylase & 0.8010 & 8 & 34.70 \\
\hline Ftsl & SSU05_1354 & Cell division protein & 0.8185 & 5 & 31.84 \\
\hline FtsQ & SSU05_0478 & Cell division septal protein & 0.8223 & 8 & 28.33 \\
\hline FtsX & SSU05_1410 & Cell division protein & 1.2542 & 6 & 24.60 \\
\hline GpsB & SSU05_0417 & Cell division protein & 1.3016 & 6 & 62.16 \\
\hline GyrA & SSU05_1267 & DNA gyrase subunit A & 0.8140 & 9 & 16.43 \\
\hline GyrB & SSU05_1510 & DNA gyrase subunit B & 0.7152 & 2 & 45.38 \\
\hline MurD & SSU05_0476 & UDP-N-acetylmuramoyl-L-alanyl-D-glutamate synthetase & 0.7718 & 14 & 39.87 \\
\hline PcrA & SSU05_0731 & Superfamily I DNA/RNA helicase & 0.8044 & 15 & 20.42 \\
\hline PhpP & SSU05_0427 & Serine/threonine protein phosphatase & 0.8067 & 11 & 59.59 \\
\hline $\mathrm{RecN}$ & SSU05_1651 & DNA repair ATPase & 0.8321 & 14 & 35.99 \\
\hline RmuC & SSU05_1991 & DNA recombination protein & 0.7751 & 8 & 19.90 \\
\hline RnhB & SSU05_0996 & Ribonuclease HII & 0.8074 & 8 & 35.41 \\
\hline RnmV & SSU05_2010 & DNA primase & 0.7144 & 6 & 26.53 \\
\hline RNase H & SSU05_0226 & Ribonuclease HIII & 0.7297 & 4 & 14.86 \\
\hline StkP & SSU05_0428 & Serine/threonine protein kinase & 0.8129 & 5 & 32.53 \\
\hline Tag & SSU05_0061 & 3-methyladenine DNA glycosylase & 0.7738 & 1 & 10.58 \\
\hline- & SSU05_0872 & Chromosome segregation ATPase & 0.7753 & 13 & 25.46 \\
\hline XerS & SSU05_0881 & Site-specific tyrosine recombinase & 0.6361 & 1 & 5.90 \\
\hline XerD & SSU05_1702 & Site-specific tyrosine recombinase XerD-like protein & 0.5529 & 3 & 15.64 \\
\hline- & SSU05_1815 & Ribonucleases G and E & 0.6327 & 6 & 6.42 \\
\hline \multicolumn{6}{|c|}{ CAPSULE SYNTHESIS } \\
\hline Cps2C & SSU05_0566 & Tyrosine-protein kinase Wze & 0.4555 & 1 & 28.57 \\
\hline Cps2F & SSU05_0569 & Rhamnosyltransferase & 1.5931 & 1 & 10.28 \\
\hline Cps2P & SSU05_0578 & Sialic acid synthase & 1.4255 & 6 & 76.63 \\
\hline Cps2Q & SSU05_0579 & UNP-N-acetyglucosamine 2-epimerase & 1.2001 & 17 & 53.58 \\
\hline Cps2R & SSU05_0580 & Acetyltransferase & 1.2131 & 2 & 44.23 \\
\hline Cps2S & SSU05_0581 & CMP-N-acetylneuraminic acid synthetase & 1.2388 & 2 & 59.47 \\
\hline \multicolumn{6}{|c|}{ VIRULENCE-ASSOCIATED PROTEINS } \\
\hline ArcA & SSU05_0624 & Arginine deiminase & 0.4071 & 15 & 43.77 \\
\hline ArcB & SSU05_0626 & Ornithine carbamoyltransferase & 0.4561 & 10 & 35.91 \\
\hline ArcC & SSU05_0627 & Carbamate kinase & 0.3703 & 4 & 23.17 \\
\hline DltA & SSU05_0638 & D-alanine-poly(phosphoribitol) ligase subunit 1 & 0.8186 & 11 & 25.24 \\
\hline Enolase & SSU05_1503 & Phosphopyruvate hydratase & 0.8146 & 26 & 72.64 \\
\hline GAPDH & SSU05_0155 & Glyceraldehyde-3-phosphate dehydrogenase & 0.7700 & 3 & 90.77 \\
\hline GlnA & SSU05_0160 & Glutamine synthetase & 0.8024 & 15 & 48.21 \\
\hline GtfA & SSU05_1555 & Glycosidase & 0.6384 & 2 & 6.22 \\
\hline IMPDH & SSU05_2183 & Inosine 5'-monophosphate dehydrogenase & 0.6649 & 1 & 45.60 \\
\hline PurA & SSU05_1966 & Adenylosuccinate synthase & 0.7722 & 22 & 60.23 \\
\hline SadP & SSU05_0272 & Translation initiation factor 2 GTPase & 0.6302 & 9 & 18.43 \\
\hline Sly & SSU05_1403 & Suilysin & 0.6552 & 4 & 11.26 \\
\hline
\end{tabular}

2014; Zhu et al., 2014; Ahmed et al., 2015; Tsang and Bernhardt, 2015). These findings can explain the mechanism by which gidA disruption inhibits $S$. suis growth. However, two cell divisionassociated proteins, GpsB and FtsX, were up-regulated. GpsB is a negative regulator of cell division by interacting with DivIVA (Fleurie et al., 2014). Thus, GpsB up-regulation can inhibit cell division of $S$. suis. FtsX together with FtsE forms a dimer that act as an ABC transporter (Schmidt et al., 2004). The FtsEX protein complex plays a major role in regulating peptidoglycan hydrolases in response to signals from cell division (Sham et al., 2013), although the precise role of FtsX in coordinating peptidoglycan hydrolases remains unknown. 
gidA deletion significantly reduced $S$. suis virulence. In vivo and ex vivo studies revealed that the mutant strain displayed reduced mortality and bacterial loads in mice, reduced ability to adhere to and invade in epithelial cells, and increased sensitivity to phagocytosis. This finding is also consistent with the regulation of virulence in A. hydrophila (Sha et al., 2004), S. enterica (Rehl et al., 2013), and P. syringae (Kinscherf and Willis, 2002). The virulence attenuation in S. suis by gidA disruption can be explained by the down-regulation of some virulence factors, including Sly, enolase, GAPDH, ADS (ArcABC), DltA, and SadP (Table 3). Sly is one of the most important virulence factors in $S$. suis, and contributes to pathogen's hemolytic activity, adhesion to and invasion of epithelial cells, host colonization, and ability to cross blood-brain barrier (Charland et al., 2000; Allen et al., 2001). The reduced hemolytic activity of $\Delta g i d A$ was confirmed by the hemolysin assay (Figure 1D). The ADS encoded by the operon $\operatorname{arc} A B C$ is also involved in ability of $S$. suis to adhere to and invade in epithelial cells (Degnan et al., 2000; Fulde et al., 2014), and to resist oxygen depletion, nutrient starvation, and acidic environments (Gruening et al., 2006). DltA is involved in D-alanylation of lipoteichoic acid which contributes to the survival of S.suis (Fittipaldi et al., 2008). In addition, enolase, GAPDH, and SadP are three important adhesins in S. suis (Ge et al., 2004).

Interestingly, the capsule of $\Delta$ gidA became much thicker than that of SC-19 (Figure 1C). This phenotype is consistent with the up-regulation of the enzymes, including Cps2F, Cps2P, Cps2Q, Cps2R, and Cps2S, which are involved in CPS synthesis (Table 3). In this study, only the tyrosine-protein kinase Cps2C was downregulated. The orthologous protein of Cps2C in Streptococcus pneumoniae $(\mathrm{CpsD})$ is a negative regulator for CPS production (Morona et al., 2003). To our best knowledge, this work is the first to report that CPS synthesis can be regulated by GidA in S. suis. CPS is considered a virulence factor in many bacteria, including S. suis (Feng et al., 2012). However, a report has suggested that the capsule only slightly contributes to the virulence of $S$. suis because both the virulent and avirulent strains can be fully encapsulated (Smith et al., 1999). Although the capsule of $\Delta$ gidA became thicker than that of the parental strain, the mutant strain was attenuated, and become more sensitive to phagocytosis. This finding is possibly affected by the use of multiple virulence factors in determination of $S$. suis virulence.

\section{REFERENCES}

Ahmed, W., Menon, S., Karthik, P. V. D. N. B., and Nagaraja, V. (2015). Autoregulation of topoisomerase I expression by supercoiling sensitive transcription. Nucleic Acids Res. 44, 1541-1552. doi: 10.1093/nar/gkv1088

Allen, A. G., Bolitho, S., Lindsay, H., Khan, S., Bryant, C., Norton, P., et al. (2001). Generation and characterization of a defined mutant of Streptococcus suis lacking suilysin. Infect. Immun. 69, 2732-2735. doi: 10.1128/IAI.69.4.27322735.2001

Brégeon, D., Colot, V., Radman, M., and Taddei, F. (2001). Translational misreading: a tRNA modification counteracts a +2 ribosomal frameshift. Genes Dev. 15, 2295-2306. doi: 10.1101/gad.207701

Charland, N., Nizet, V., Rubens, C. E., Kim, K. S., Lacouture, S., and Gottschalk, M. (2000). Streptococcus suis serotype 2 interactions with human
There are two technical issues needed to be discussed here. The first one is about the complementary strain. We failed to create a complementary strain of the mutant (in most cases it is difficult for S. suis). Therefore we have performed the RTPCR to exclude polarity effect. In addition, identical phenotypes were observed with independently-obtained gidA mutants. In our view, this fact is sufficient to rule out the remaining possibility of distant secondary mutations whose chance to occur at the same place in two independent gidA mutants is extremely low. The second issue is about iTRAQ. Concerning that GidA can affect the accuracy of protein translation, truncated, or mistranslated proteins may be produced in the gidA mutant. These proteins may not be detected by iTRAQ. The information of this part of proteins was ignored in this study.

In conclusion, our data suggest that the tRNA modification enzyme GidA is a translational regulator for the expression of particular proteins involved in the growth, cell division, capsule synthesis, and virulence of the zoonotic S. suis. These findings provide a new insight that lead to our better understanding of the regulatory function of GidA in bacterial pathogens.

\section{AUTHOR CONTRIBUTIONS}

The experiments were performed mainly by TG, MT, and WL, and some experiments were performed with the assistance of $\mathrm{CZ}$, JZ, and LZ. TG, LL, and TZ analyzed the data. The study was designed by RZ. TG and RZ wrote the manuscript.

\section{ACKNOWLEDGMENTS}

This work was supported by the National Basic Research Program of China (973 Program grant No. 2012CB518802) and the International S \& T Cooperation Program of China (ISTCP grant No. 2013DFG32360). We are grateful to Dr. Yosuke Murakami for providing us the pSET plasmids.

\section{SUPPLEMENTARY MATERIAL}

The Supplementary Material for this article can be found online at: http://journal.frontiersin.org/article/10.3389/fcimb. 2016.00044

brain microvascular endothelial cells. Infect. Immun. 68, 637-643. doi: 10.1128/IAI.68.2.637-643.2000

Degnan, B. A., Fontaine, M. C., Doebereiner, A. H., Lee, J. J., Mastroeni, P., Dougan, G., et al. (2000). Characterization of an isogenic mutant of Streptococcus pyogenes Manfredo lacking the ability to make streptococcal acid glycoprotein. Infect. Immun. 68, 2441-2448. doi: 10.1128/IAI.68.5.24412448.2000

Dinardo, S., Voelkel, K. A., Sternglanz, R., Reynolds, A. E., and Wright, A. (1982). Escherichia coli DNA topoisomerase I mutants have compensatory mutations in DNA gyrase genes. Cell 31, 43-51. doi: 10.1016/0092-8674(82)9 0403-2

Edwards, D. H., and Errington, J. (1997). The Bacillus subtilis DivIVA protein targets to the division septum and controls the site specificity of cell division. Mol. Microbiol. 24, 905-915. doi: 10.1046/j.1365-2958.1997.3811764.x 
Feng, Y., Cao, M., Shi, J., Zhang, H., Hu, D., Zhu, J., et al. (2012). Attenuation of Streptococcus suis virulence by the alteration of bacterial surface architecture. Sci. Rep. 2:710. doi: 10.1038/srep00710

Feng, Y. J., Zhang, H. M., Wu, Z. W., Wang, S. H., Cao, M., Hu, D., et al. (2014). Streptococcus suis infection an emerging/reemerging challenge of bacterial infectious diseases? Virulence 5, 477-497. doi: 10.4161/viru.28595

Ferrando, M. L., Van Baarlen, P., Orrù, G., Piga, R., Bongers, R. S., Wels, M., et al. (2014). Carbohydrate availability regulates virulence gene expression in Streptococcus suis. PLoS ONE 9:e89334. doi: 10.1371/journal.pone.0089334

Ferreira, H., Butler-Cole, B., Burgin, A., Baker, R., Sherratt, D. J., and Arciszewska, L. K. (2003). Functional analysis of the C-terminal domains of the site-specific recombinases XerC and XerD. J. Mol. Biol. 330, 15-27. doi: 10.1016/S00222836(03)00558-8

Fislage, M., Brosens, E., Deyaert, E., Spilotros, A., Pardon, E., Loris, R., et al. (2014). SAXS analysis of the tRNA-modifying enzyme complex MnmE/MnmG reveals a novel interaction mode and GTP-induced oligomerization. Nucleic Acids Res. 42, 5978-5992. doi: 10.1093/nar/gku213

Fittipaldi, N., Segura, M., Grenier, D., and Gottschalk, M. (2012). Virulence factors involved in the pathogenesis of the infection caused by the swine pathogen and zoonotic agent Streptococcus suis. Future Microbiol. 7, 259-279. doi: $10.2217 /$ fmb.11.149

Fittipaldi, N., Sekizaki, T., Takamatsu, D., Harel, J., Domínguez-Punaro Mde, L., Von Aulock, S., et al. (2008). D-alanylation of lipoteichoic acid contributes to the virulence of Streptococcus suis. Infect. Immun. 76, 3587-3594. doi: 10.1128/IAI.01568-07

Fleurie, A., Manuse, S., Zhao, C., Campo, N., Cluzel, C., Lavergne, J. P., et al. (2014). Interplay of the serine/threonine-kinase StkP and the paralogs DivIVA and GpsB in pneumococcal cell elongation and division. PLoS Genet. 10:e1004275. doi: 10.1371/journal.pgen.1004275

Fulde, M., Willenborg, J., Huber, C., Hitzmann, A., Willms, D., Seitz, M., et al. (2014). The arginine-ornithine antiporter ArcD contributes to biological fitness of Streptococcus suis. Front. Cell. Infect. Microbiol. 4:107. doi: 10.3389/fcimb.2014.00107

Ge, J. P., Catt, D. M., and Gregory, R. L. (2004). Streptococcus mutans surface alphaenolase binds salivary mucin MG2 and human plasminogen. Infect. Immun. 72, 6748-6752. doi: 10.1128/IAI.72.11.6748-6752.2004

Gruening, P., Fulde, M., Valentin-Weigand, P., and Goethe, R. (2006). Structure, regulation, and putative function of the arginine deiminase system of Streptococcus suis. J. Bacteriol. 188, 361-369. doi: 10.1128/JB.188.2.361369.2006

Jacobs, A. A., Loeffen, P. L., Van Den Berg, A. J., and Storm, P. K. (1994). Identification, purification, and characterization of a thiol-activated hemolysin (suilysin) of Streptococcus suis. Infect. Immun. 62, 1742-1748.

Janto, B., Ahmed, A., Ito, M., Liu, J., Hicks, D. B., Pagni, S., et al. (2011). Genome of alkaliphilic Bacillus pseudofirmus OF4 reveals adaptations that support the ability to grow in an external $\mathrm{pH}$ range from 7.5 to 11.4. Environ. Microbiol. 13, 3289-3309. doi: 10.1111/j.1462-2920.2011.02591.x

Jing, H. B., Yuan, J., Wang, J., Yuan, Y., Zhu, L., Liu, X. K., et al. (2008). Proteome analysis of Streptococcus suis serotype 2. Proteomics 8, 333-349. doi: 10.1002/pmic.200600930

Kinscherf, T. G., and Willis, D. K. (2002). Global regulation by gidA in Pseudomonas syringae. J. Bacteriol. 184, 2281-2286. doi: 10.1128/JB.184.8.22812286.2002

Li, D., Shibata, Y., Takeshita, T., and Yamashita, Y. (2014). A novel gene involved in the survival of Streptococcus mutans under stress conditions. Appl. Environ. Microbiol. 80, 97-103. doi: 10.1128/AEM.02549-13

Li, W., Hu, X., Liu, L., Chen, H., and Zhou, R. (2011). Induction of protective immune response against Streptococcus suis serotype 2 infection by the surface antigen HP0245. FEMS Microbiol. Lett. 316, 115-122. doi: 10.1111/j.15746968.2010.02200.x

Li, W., Liu, L., Chen, H., and Zhou, R. (2009). Identification of Streptococcus suis genes preferentially expressed under iron starvation by selective capture of transcribed sequences. FEMS Microbiol. Lett. 292, 123-133. doi: 10.1111/j.15746968.2008.01476.x

Li, W., Liu, L., Qiu, D., Chen, H., and Zhou, R. (2010). Identification of Streptococcus suis serotype 2 genes preferentially expressed in the natural host. Int. J. Med. Microbiol. 300, 482-488. doi: 10.1016/j.ijmm.2010.04.018
Li, W., Wan, Y., Tao, Z., Chen, H., and Zhou, R. (2013). A novel fibronectinbinding protein of Streptococcus suis serotype 2 contributes to epithelial cell invasion and in vivo dissemination. Vet. Microbiol. 162, 186-194. doi: 10.1016/j.vetmic.2012.09.004

Lun, Z. R., Wang, Q. P., Chen, X. G., Li, A. X., and Zhu, X. Q. (2007). Streptococcus suis: an emerging zoonotic pathogen. Lancet Infect. Dis. 7, 201-209. doi: 10.1016/S1473-3099(07)70001-4

Marion, C., Aten, A. E., Woodiga, S. A., and King, S. J. (2011). Identification of an ATPase, MsmK, which energizes multiple carbohydrate ABC transporters in Streptococcus pneumoniae. Infect. Immun. 79, 4193-4200. doi: 10.1128/IAI.05290-11

Morona, J. K., Morona, R., Miller, D. C., and Paton, J. C. (2003). Mutational analysis of the carboxy-terminal (YGX)4 repeat domain of $\mathrm{CpsD}$, an autophosphorylating tyrosine kinase required for capsule biosynthesis in Streptococcus pneumoniae. J. Bacteriol. 185, 3009-3019. doi: 10.1128/JB.185.10.3009-3019.2003

Rehl, J. M., Shippy, D. C., Eakley, N. M., Brevik, M. D., Sand, J. M., Cook, M. E., et al. (2013). GidA expression in Salmonella is modulated under certain environmental conditions. Curr. Microbiol. 67, 279-285. doi: 10.1007/s00284013-0361-2

Sandberg, A., Lindell, G., Källström, B. N., Branca, R. M., Danielsson, K. G., Dahlberg, M., et al. (2012). Tumor proteomics by multivariate analysis on individual pathway data for characterization of vulvar cancer phenotypes. Mol. Cell. Proteomics 11:M112.016998. doi: 10.1074/mcp.M112.016998

Schmidt, K. L., Peterson, N. D., Kustusch, R. J., Wissel, M. C., Graham, B., Phillips, G. J., et al. (2004). A predicted ABC transporter, FtsEX, is needed for cell division in Escherichia coli. J. Bacteriol. 186, 785-793. doi: 10.1128/JB.186.3.785-793.2004

Sha, J., Kozlova, E. V., Fadl, A. A., Olano, J. P., Houston, C. W., Peterson, J. W., et al. (2004). Molecular characterization of a glucose-inhibited division gene, gidA, that regulates cytotoxic enterotoxin of Aeromonas hydrophila. Infect. Immun. 72, 1084-1095. doi: 10.1128/IAI.72.2.1084-1095.2004

Sham, L. T., Jensen, K. R., Bruce, K. E., and Winkler, M. E. (2013). Involvement of FtsE ATPase and FtsX extracellular loops 1 and 2 in FtsEX-PcsB complex function in cell division of Streptococcus pneumoniae D39. Mbio 4:e00431-13. doi: $10.1128 / \mathrm{mBio} .00431-13$

Shi, R., Villarroya, M., Ruiz-Partida, R., Li, Y., Proteau, A., Prado, S., et al. (2009). Structure-function analysis of Escherichia coli MnmG (GidA), a highly conserved tRNA-modifying enzyme. J. Bacteriol. 191, 7614-7619. doi: 10.1128/JB.00650-09

Shippy, D. C., Eakley, N. M., Bochsler, P. N., Chopra, A. K., and Fadl, A. A. (2011). Biological and virulence characteristics of Salmonella enterica serovar Typhimurium following deletion of glucose-inhibited division (gidA) gene. Microb. Pathog. 50, 303-313. doi: 10.1016/j.micpath.2011.02.004

Shippy, D. C., Heintz, J. A., Albrecht, R. M., Eakley, N. M., Chopra, A. K., and Fadl, A. A. (2012). Deletion of glucose-inhibited division (gidA) gene alters the morphological and replication characteristics of Salmonella enterica Serovar typhimurium. Arch. Microbiol. 194, 405-412. doi: 10.1007/s00203-011-0769-7

Šink, R., Barreteau, H., Patin, D., Mengin-Lecreulx, D., Gobec, S., and Blanot, D. (2013). MurD enzymes: some recent developments. Biomol. Concepts 4, 539-556. doi: 10.1515/bmc-2013-0024

Smith, H. E., Damman, M., Van Der Velde, J., Wagenaar, F., Wisselink, H. J., Stockhofe-Zurwieden, N., et al. (1999). Identification and characterization of the cps locus of Streptococcus suis serotype 2: the capsule protects against phagocytosis and is an important virulence factor. Infect. Immun. 67, 1750-1756.

Takamatsu, D., Osaki, M., and Sekizaki, T. (2001). Thermosensitive suicide vectors for gene replacement in Streptococcus suis. Plasmid 46, 140-148. doi: 10.1006/plas.2001.1532

Tan, M. F., Gao, T., Liu, W. Q., Zhang, C. Y., Yang, X., Zhu, J. W., et al. (2015). MsmK, an ATPase, contributes to utilization of multiple carbohydrates and host colonization of Streptococcus suis. PLoS ONE 10:e0130792. doi: 10.1371/journal.pone.0130792

Trieu-Cuot, P., Carlier, C., Poyart-Salmeron, C., and Courvalin, P. (1991). Shuttle vectors containing a multiple cloning site and a lacZ alpha gene for conjugal transfer of DNA from Escherichia coli to gram-positive bacteria. Gene 102, 99-104. doi: 10.1016/0378-1119(91)90546-N 
Tsang, M. J., and Bernhardt, T. G. (2015). A role for the FtsQLB complex in cytokinetic ring activation revealed by an $\mathrm{ftsL}$ allele that accelerates division. Mol. Microbiol. 95, 925-944. doi: 10.1111/mmi.12905

Van De Rijn, I., and Kessler, R. E. (1980). Growth characteristics of group A streptococci in a new chemically defined medium. Infect. Immun. 27, 444-448.

Von Meyenburg, K., Jørgensen, B. B., Nielsen, J., and Hansen, F. G. (1982). Promoters of the atp operon coding for the membrane-bound ATP synthase of Escherichia coli mapped by Tn10 insertion mutations. Mol. Gen. Genet. 188, 240-248. doi: 10.1007/BF00332682

Wertheim, H. F., Nghia, H. D., Taylor, W., and Schultsz, C. (2009). Streptococcus suis: an emerging human pathogen. Clin. Infect. Dis. 48, 617-625. doi: $10.1086 / 596763$

Yim, L., Moukadiri, I., Björk, G. R., and Armengod, M. E. (2006). Further insights into the tRNA modification process controlled by proteins $\mathrm{MnmE}$ and GidA of Escherichia coli. Nucleic Acids Res. 34, 5892-5905. doi: 10.1093/nar/gkl752

Yu, H., Jing, H., Chen, Z., Zheng, H., Zhu, X., Wang, H., et al. (2006). Human Streptococcus suis outbreak, Sichuan, China. Emerging Infect. Dis. 12, 914-920. doi: 10.3201/eid1206.051194

Zhang, T., Ding, Y., Li, T., Wan, Y., Li, W., Chen, H., et al. (2012). A Fur-like protein PerR regulates two oxidative stress response related operons dpr and metQIN in Streptococcus suis. BMC Microbiol. 12:85. doi: 10.1186/1471-218 $0-12-85$
Zhang, W., Zhao, Z., Zhang, B., Wu, X. G., Ren, Z. G., and Zhang, L. Q. (2014). Posttranscriptional regulation of 2,4-diacetylphloroglucinol production by GidA and TrmE in Pseudomonas fluorescens 2P24. Appl. Environ. Microbiol. 80, 3972-3981. doi: 10.1128/AEM.00455-14

Zheng, F., Ji, H., Cao, M., Wang, C., Feng, Y., Li, M., et al. (2011). Contribution of the Rgg transcription regulator to metabolism and virulence of Streptococcus suis serotype 2. Infect. Immun. 79, 1319-1328. doi: 10.1128/IAI.00193-10

Zhu, H., Zhou, J., Ni, Y., Yu, Z., Mao, A., Hu, Y., et al. (2014). Contribution of eukaryotic-type serine/threonine kinase to stress response and virulence of Streptococcus suis. PLoS ONE 9:e91971. doi: 10.1371/journal.pone.0091971

Conflict of Interest Statement: The authors declare that the research was conducted in the absence of any commercial or financial relationships that could be construed as a potential conflict of interest.

Copyright $\odot 2016$ Gao, Tan, Liu, Zhang, Zhang, Zheng, Zhu, Li and Zhou. This is an open-access article distributed under the terms of the Creative Commons Attribution License (CC BY). The use, distribution or reproduction in other forums is permitted, provided the original author(s) or licensor are credited and that the original publication in this journal is cited, in accordance with accepted academic practice. No use, distribution or reproduction is permitted which does not comply with these terms. 